\title{
Otimização da produção de hidrogênio utilizando o algoritmo genético
}

Optimization of hydrogen production by using genetic algorithm

\author{
L. D. de S. Netto ${ }^{1}$; T. V. G. Reis ${ }^{2}$; A. da S. Guimarães ${ }^{2}$; P. L. de Santana ${ }^{1}$; A. S. \\ Silva ${ }^{1}$; R. L. Pagano, \\ ${ }^{1}$ Programa de pós-graduação em Engenharia Química /Laboratório de Modelagem e Simulação/CCET, Universidade \\ Federal de Sergipe, 49100-000, São Cristóvão/SE, Brasil. \\ ${ }^{2}$ Departamento de Engenharia Química /Laboratório de Modelagem e Simulação/CCET, Universidade Federal de \\ Sergipe, 49100-000, São Cristóvão/SE, Brasil. \\ *rpagano@ufs.br
}

(Recebido em 14 de março de 2016; aceito em 10 de abril de 2016)

\begin{abstract}
A pesquisa por novas alternativas e processos geradores de energia tornou-se fundamental para atender à demanda mundial por fontes energéticas sustentáveis e menos agressivas ao meio ambiente. Defronte destes fatos, a produção de hidrogênio converte-se numa alternativa viável, por ser considerado um combustível limpo e de alta densidade energética. A principal rota industrial para a obtenção de hidrogênio é a reforma a vapor do metano. Este é um processo endotérmico, no qual o metano reage com o vapor d'água, sob condições de elevadas temperaturas e pressão, para geração de hidrogênio. Recentemente, os avanços na área de modelagem e simulação, especialmente aplicando a técnica de fluidodinâmica computacional, vem auxiliando na investigação e otimização destes processos, sem que haja gastos relacionados à realização do mesmo. Diante deste contexto, o presente trabalho teve como finalidade simular um reator com membrana por meio de um software comercial, com a finalidade de realizar a otimização das suas condições operacionais. O Algoritmo Genético (AG) foi aplicado com o intuito de maximizar a produtividade do processo. Foram obtidos perfis simulados do reator para a conversão de metano e recuperação de hidrogênio alcançando valores de $100 \%$, o que comprova a eficácia da metodologia apresentada para otimizar as principais variáveis do processo.
\end{abstract}

Palavras-chave: Reforma a vapor, CFD, Algoritmo Genético

The research for new alternatives and energy generating processes has become crucial to understand the global demand for sustainable and less aggressive energy sources to the environment. Facing these facts, the production of hydrogen becomes a viable alternative, since it is considered a clean and high density energy fuel. The main industrial route for obtaining hydrogen is the steam methane reforming. This is an endothermic process, in which methane reacts with steam at high temperature and pressure conditions to yield hydrogen. Recently, advances in modeling and simulation field, especially applying computational fluid dynamics technique, is aiding in the investigation and optimization of these processes, with low expenses. In this context, this study aimed to simulate a reactor with membrane by applying commercial software, in order to perform the optimization of its operational conditions. The Genetic Algorithm (GA) was applied in order to maximize the productivity of the process. Simulated profiles of the reactor for the conversion of methane and hydrogen recovery were obtained reaching values of $100 \%$, which proves the effectiveness of the methodology presented for optimizing the main process variables.

Keywords: Steam reforming, CFD, Genetic Algorithm

\subsection{INTRODUÇÃO}

O hidrogênio é constantemente referido como fonte de energia limpa, pois a sua combustão produz apenas água e calor. Segundo Nicodemos [1], o hidrogênio pode ser considerado o combustível do futuro, esta designação deve-se ao fato do hidrogênio ser o mais abundante elemento da tabela periódica, além de possuir um elevado valor energético e alta reatividade, bem como ser um elemento não tóxico.

Atualmente, a principal rota industrial de produção de hidrogênio é a reforma a vapor do metano, isto se deve às vantagens econômicas que este processo apresenta em relação a outros processos de produção de hidrogênio. Além disso, as grandes reservas de gás natural disponíveis 
no mundo tornam este processo de reforma uma solução promissora e economicamente viável para produção de hidrogênio e gás de síntese [2].

A reforma a vapor do metano abrange duas etapas de reação: na primeira, o metano reage com o vapor d'água para gerar gás hidrogênio e monóxido de carbono (gás de síntese), sob condições de temperatura e pressão elevadas (Equação 1), e uma segunda reação denominada deslocamento gás-d'água, na qual se alterna o uso de diferentes temperaturas para melhorar a conversão dos reagentes em produtos (Equação 2), esta etapa visa aumentar a eficiência do processo. Por fim, a terceira reação representa a reação global entre as duas (Equação 3) [3].

$$
\begin{array}{ll}
\mathrm{CH}_{4}+\mathrm{H}_{2} \mathrm{O} \leftrightarrow \mathrm{CO}+3 \mathrm{H}_{2} & \Delta \mathrm{H}_{298}^{0}=206 \mathrm{~kJ} \cdot \mathrm{mol}^{-1} \\
\mathrm{CO}+\mathrm{H}_{2} \mathrm{O} \leftrightarrow \mathrm{CO}_{2}+\mathrm{H}_{2} & \Delta \mathrm{H}_{298}^{0}=-41 \mathrm{~kJ} \cdot \mathrm{mol}^{-1} \\
\mathrm{CH}_{4}+2 \mathrm{H}_{2} \mathrm{O} \leftrightarrow \mathrm{CO}_{2}+4 \mathrm{H}_{2} & \Delta \mathrm{H}_{298}^{\circ}=165 \mathrm{~kJ} \cdot \mathrm{mol}^{-1}
\end{array}
$$

O gás de síntese produzido no processo de reforma deve ser separado, com o objetivo de produzir hidrogênio de alta pureza. Os reatores com membrana podem ser aplicados para esta finalidade, além de proporcionar diversas vantagens ao processo. Dentre as vantagens mencionadas, estão a redução do volume de operação e uma maior conversão dos reagentes, devido ao deslocamento do equilíbrio termodinâmico da reação em favor dos produtos [4]. O uso deste tipo de recurso distingue-se também de outros processos, por contribuir para a utilização de condições operacionais mais amenas.

Devido a elevados custos operacionais que envolvem a realização do processo de reforma, a fluidodinâmica computacional pode ser aplicada como ferramenta para o estudo da influência das diferentes variáveis do processo, bem como na descrição e investigação do mesmo. De uma forma geral, estudos teóricos de modelagem, simulação e otimização podem auxiliar na busca de melhores condições operacionais, sendo de grande importância para o desenvolvimento de pesquisas na área. Dentre os métodos de otimização determinísticos e heurísticos conhecidos, neste trabalho, foi utilizado o método conhecido como Algoritmo Genético (AG), classificado com um método heurístico, o qual é baseado na teoria da evolução das espécies de Darwin aplicando o processo de seleção natural. Conforme esta teoria, os indivíduos que se adaptam melhor ao meio ambiente, terão maiores chances de sobreviver e de passar seus genes para as próximas gerações, assegurando a evolução contínua da espécie. Além disso, a ocorrência de mutações permite a ocorrência de saltos na evolução das espécies [5-9].

Neste contexto, este trabalho objetivou simular um reator com membrana por meio de um software comercial, a fim de otimizá-lo com o objetivo de se obter condições operacionais ótimas para o processo utilizando o método heurístico de otimização conhecido como Algoritmo Genético.

\section{MATERIAIS E MÉTODOS}

\subsection{MODELAGEM MATEMÁTICA E SIMULAÇÃO COMPUTACIONAL}

O reator com membrana simulado neste trabalho para produção de hidrogênio foi baseado nos experimentos de Shu et al. [10], com reações e equações descritas por Xu e Froment [11]. A simulação deste reator foi realizada utilizando o pacote de fluidodinâmica computacional da ANSYS Technology 14. A etapa inicial da simulação foi a criação da geometria do mesmo com o auxílio do DesignModeler do software CFX. As dimensões físicas, propostas por Shu et al. [10], utilizadas na geometria do reator estão listadas na Tabela 1.

Para esta simulação foi criada uma geometria que corresponde ao segmento circular de um cilindro com ângulo central de $15^{\circ}$, o qual representa uma parte do reator tubular descrito por Shu et al. [10]. Esta configuração foi adotada com o intuito de diminuir o tempo computacional gasto pelo software para solucionar o problema. Pode-se visualizar a geometria criada na Figura 1. 
Tabela 1: Características geométricas e operacionais do reator com membrana

\begin{tabular}{cc}
\hline Área da membrana & $10,40 \cdot 10^{-4} \mathrm{~m}^{2}$ \\
\hline Espessura da membrana & $1,98 \cdot 10^{-4} \mathrm{~m}$ \\
\hline Área da seção transversal & $2,32 \cdot 10^{-4} \mathrm{~m}^{2}$ \\
\hline $\begin{array}{c}\text { Área de troca térmica entre o } \\
\text { meio reacional e o meio externo }\end{array}$ & $62,83 \cdot 10^{-4} \mathrm{~m}^{2}$ \\
\hline Comprimento do reator & $3,60 \cdot 10^{-4} \mathrm{~m}$ \\
\hline
\end{tabular}

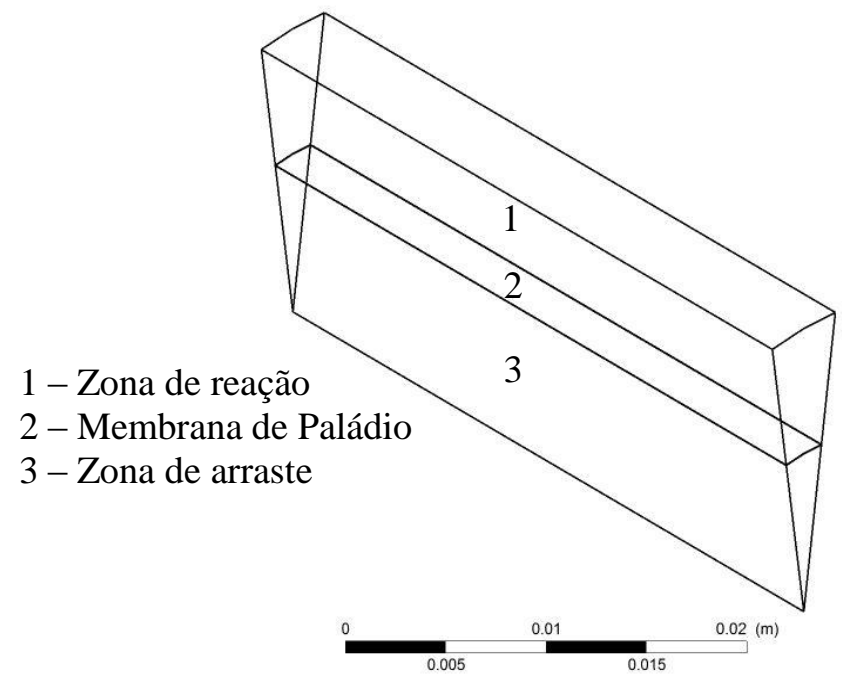

Figura 1: Geometria do reator com membrana

Para a geração da malha, foi utilizado o aplicativo Mesh. Nesse aplicativo, a geometria criada no passo anterior é carregada, as configurações da malha a serem utilizadas foram inseridas e as faces e domínios foram nomeados. Foi realizado o estudo de convergência de malha de acordo com a metodologia proposta por Roache [12], o qual será mais bem descrito na seção Estudo de Convergência de Malha.

$\mathrm{Na}$ etapa seguinte, após a geração da malha, foram inseridas as configurações do processo a serem simuladas. Para isto, o aplicativo Setup foi utilizado. Foi adotada a cinética de reação proposta por Xu e Froment [11] para descrever as reações de reforma e deslocamento gás-água.

\subsubsection{Taxas de reações das espécies}

A cinética de $\mathrm{Xu}$ e Froment [11] foi determinada para um catalisador de Ni suportado em $\mathrm{MgAl}_{2} \mathrm{O}_{4}$. Baseando-se no mecanismo de Langmuir-Hinshelwood, as taxas das reações das Equações 1, 2 e 3 são representadas pelas Equações 4, 5 e 6, respectivamente.

$r_{1}=\frac{\frac{k_{1}}{p_{H_{2}}^{2,5}}\left(p_{\left.C_{H_{4}} p_{H_{2} O}-\frac{p_{H_{2}}^{3} p_{C O}}{K_{a, 1}}\right)}\right.}{(D E N)^{2}}$

$r_{2}=\frac{\frac{k_{2}}{p_{H_{2}}}\left(p_{C O} p_{\mathrm{H}_{2} O}-\frac{p_{\mathrm{H}_{2}} p_{C O}}{K_{a, 2}}\right)}{(D E N)^{2}}$

$r_{3}=\frac{\frac{k_{3}}{p_{H_{2}}^{3,5}}\left(p_{\mathrm{CH}_{4}} p_{\mathrm{H}_{2} \mathrm{O}^{2}}^{2}-\frac{p_{\mathrm{H}_{2}}^{4} p_{\mathrm{CO}_{2}}}{K_{a, 3}}\right)}{(D E N)^{2}}$

sendo 
$D E N=1+K_{C O} p_{C O}+K_{H_{2}} p_{H_{2}}+K_{C_{4}} p_{C_{4}}+\frac{K_{H_{2} O} p_{H_{2} O}}{p_{H_{2}}}$

com $p_{j}\left(\mathrm{j}=\mathrm{CH}_{4}, \mathrm{H}_{2} \mathrm{O}, \mathrm{CO}_{2}, \mathrm{CO}\right.$ e $\left.\mathrm{H}_{2}\right)$ a pressão parcial de cada espécie, $k_{i}$ a constante da taxa de reação i (i $=1,2$ e 3$), K_{i}$ a constante de equilíbrio da reação i e $K_{a, j}$ a constante de adsorção de cada espécie, e as expressões para tais constantes são descritas pelas Equações 8, 9 e 10, respectivamente.

$k_{i}=A\left(k_{i}\right) \exp \left(\frac{-E_{i}}{R T}\right)$

$K_{i}=A\left(K_{i}\right) \exp \left(\frac{-\Delta H_{i}}{R T}\right)$

$K_{a, j}=A\left(K_{j}\right) \exp \left(\frac{-\Delta H_{a, j}}{R T}\right)$

onde $E_{i}$ é a energia de ativação da reação i, $\Delta H_{i}$ a variação de entalpia de reação da reação i, $\Delta H_{a, j}$ a variação de entalpia de adsorção da espécie $\mathrm{j}$ todos em $\mathrm{J} \cdot \mathrm{mol}^{-1}, \mathrm{R}$ a constante dos gases ideais $\left(\mathrm{J} \cdot \mathrm{mol}^{-1} \cdot \mathrm{K}^{-1}\right)$ e T a temperatura da reação $(\mathrm{K})$.

As equações das taxas de reação para cada espécie no reator de membrana são dadas através das Equações 11, 12, 13, 14 e 15, com base nas expressões de taxa de reação das duas reações de reforma a vapor (Equações 1 e 3) e a na reação de deslocamento gás-água (Equação 2).

$$
\begin{aligned}
& r_{\mathrm{CH}_{4}}=-\left(r_{1}+r_{3}\right) \\
& r_{\mathrm{CO}_{2}}=r_{2}+r_{3} \\
& r_{\mathrm{CO}}=r_{1}-r_{2} \\
& r_{\mathrm{H}_{2} \mathrm{O}}=-\left(r_{1}+r_{2}+2 r_{3}\right) \\
& r_{\mathrm{H}_{2}}=3 r_{1}+r_{2}+4 r_{3}
\end{aligned}
$$

\subsubsection{Modelagem}

As hipóteses adotadas para a modelagem matemática foram as seguintes: sistema de coordenadas cilíndricas, escoamento em regime permanente, tridimensional e turbulento; fluido compressível; sistema isotérmico; e sistema com geração e consumo de espécies. As equações de balanço de massa (Equação 16) e quantidade de movimento (Equação 17) são as equações utilizadas pelo pacote computacional $A N S Y S / C F X^{\circledR}$ versão 14 . O pacote computacional utiliza o método de volume finitos para solucionar o problema, que se baseia em dividir o domínio computacional em pequenos volumes de controles e, em seguida, integra temporalmente e espacialmente cada equação de transporte em cada volume de controle, estabelecendo uma equação discreta que expresse a mesma lei de conservação em cada um deles. Como resultado, uma aproximação do valor de cada variável é obtida, em pontos específicos através do domínio [13]. Desta forma, pode-se obter um perfil tridimensional do comportamento do escoamento. O modelo de turbulência usado para a zona de reação e zona de arraste foi o SSG Reynolds Stress. A permeação do hidrogênio é descrita pela lei Sieverts-Fick (Equação 18). A porosidade do leito catalítico foi calculada através da expressão desenvolvida por Reichelt e Blasz [14] (Equação 19). O cálculo da produtividade do processo foi descrito pela Equação 20.

$\nabla \cdot(\rho \vec{v})=S_{m}$

$\nabla \cdot(\rho \vec{v} \vec{v})=-\nabla P+\nabla:\left(\mu\left[\left(\nabla \vec{v}+\nabla \vec{v}^{T}\right)-\frac{2}{3} \nabla \cdot \vec{v} I\right]\right)+\rho \vec{g}+\vec{F}$

onde $\rho$ é a densidade do fluido $\left(\mathrm{kg} \cdot \mathrm{m}^{-3}\right), \vec{v}$ vetor velocidade do fluido $\left(\mathrm{m} \cdot \mathrm{s}^{-1}\right), S_{m}$ é o termo volumétrico de geração e/ou consumo de massa por unidade de volume $\left(\mathrm{kg} \cdot \mathrm{m}^{-3} \cdot \mathrm{s}^{-1}\right), \mathrm{P}$ é a pressão estática (Pa), $\mu$ é a viscosidade do fluido $(\mathrm{Pa} \cdot \mathrm{s}), \vec{g}$ é a aceleração da gravidade $\left(\mathrm{m} \cdot \mathrm{s}^{-2}\right), I$ é o tensor unitário e $\vec{F}$ é a força aplicada sobre o fluido (N). 


$$
\begin{aligned}
& J_{H_{2}}=P e_{H_{2}}^{0} \exp \left(-\frac{E_{a}}{R T}\right) \cdot \frac{\left(p_{H_{2}, \text { retido }}^{0,5}-p_{H_{2}, \text { permeado }}^{0,5}\right)}{\delta} \\
& \varepsilon_{B}=0,38+0,073 \cdot\left[1+\frac{\left(\frac{D_{r}}{d_{p}}-2\right)^{2}}{\left(\frac{D_{r}}{d_{p}}\right)^{2}}\right] \\
& \text { Prod }=\frac{x_{\mathrm{H}_{2}}^{\text {permeado }} \cdot f_{\text {arraste }}}{x_{\mathrm{CH} H_{4}}^{\text {inicial }} \cdot f_{\text {reação }}}
\end{aligned}
$$

sendo $P e_{H_{2}}^{0}$ o fator pre-exponencial da permeabilidade do hidrogênio $\left(\mathrm{mol} \cdot \mathrm{m}^{-1} \cdot \mathrm{s}^{-1} \cdot \mathrm{Pa}^{-0,5}\right), E_{a}$ a energia de ativação da membrana $\left(\mathrm{J} \cdot \mathrm{mol}^{-1}\right), p_{H_{2}}$,retido a pressão parcial de hidrogênio na reação $(\mathrm{Pa}), p_{H_{2}}$,permeado a pressão parcial de hidrogênio no arraste $(\mathrm{Pa}), \delta$ a espessura da membrana $(\mathrm{m}), \varepsilon_{B}$ a porosidade do leio catalítico, $D_{r}$ a diferença entre o diâmetro interno do tubo externo do reator e o diâmetro externo do tubo interno do reator $(\mathrm{m}), d_{p}$ é o diâmetro do poro do leito catalítico $(\mathrm{m})$, farraste e $f_{\text {reação }}$ são o fluxo mássico no arraste e reação $\left(\mathrm{kg} \cdot \mathrm{m}^{-2} \cdot \mathrm{s}^{-1}\right)$,

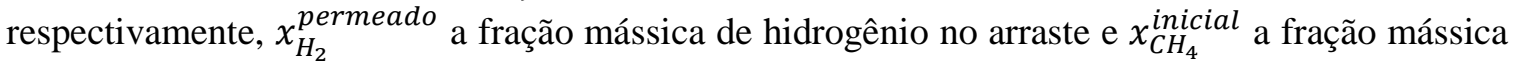
inicial de metano.

Os valores dos fatores pré-exponenciais, energias de ativação e variação de entalpias utilizados nesta modelagem estão apresentados na Tabela 2.

Tabela 2: Constantes e parâmetros cinéticos utilizados no modelo

\begin{tabular}{cccc}
\hline Parâmetros & $\begin{array}{c}\text { Fatores } \\
\text { pré-exponencial }\end{array}$ & $\begin{array}{c}\text { Unidades dos } \\
\text { Fatores pré-exponenciais }\end{array}$ & $\begin{array}{c}\mathbf{E} \text { e } \mathbf{\Delta H} \\
(\mathbf{k J} / \mathbf{m o l})\end{array}$ \\
\hline $\boldsymbol{k}_{\mathbf{1}}$ & $1,34 \cdot 10^{15}$ & $\mathrm{~mol} \cdot \mathrm{MPa}^{0,5} \cdot \mathrm{kg}_{\mathrm{cat}}^{-1} \cdot \mathrm{h}^{-1}$ & 240,10 \\
\hline $\boldsymbol{K}_{\mathbf{2}}$ & $1,96 \cdot 10^{7}$ & $\mathrm{~mol} \cdot \mathrm{kg}_{\mathrm{cat}}^{-1} \cdot \mathrm{h}^{-1} \cdot \mathrm{Mpa}^{-1}$ & 67,13 \\
\hline $\boldsymbol{k}_{\mathbf{3}}$ & $3,23 \cdot 10^{14}$ & $\mathrm{~mol} \cdot \mathrm{MPa}^{0,5} \cdot \mathrm{kg}_{\mathrm{cat}}{ }^{-1} \cdot \mathrm{h}^{-1}$ & 243,90 \\
\hline $\boldsymbol{K}_{\mathbf{1}}$ & $8,06 \cdot 10^{22}$ & $\mathrm{~Pa}^{2}$ & 220,20 \\
\hline $\boldsymbol{K}_{\mathbf{2}}$ & $1,41 \cdot 10^{-2}$ & - & $-37,72$ \\
\hline $\boldsymbol{K}_{\mathbf{3}}$ & $1,14 \cdot 10^{21}$ & $\mathrm{~Pa}^{2}$ & 182,40 \\
\hline $\boldsymbol{K}_{\boldsymbol{C} \boldsymbol{H}_{\mathbf{4}}}$ & $6,65 \cdot 10^{-3}$ & $\mathrm{MPa}^{-1}$ & $-38,28$ \\
\hline $\boldsymbol{K}_{\boldsymbol{C} \boldsymbol{O}}$ & $8,23 \cdot 10^{-4}$ & $\mathrm{MPa}^{-1}$ & $-70,65$ \\
\hline $\boldsymbol{K}_{\boldsymbol{H}_{\mathbf{2}} \boldsymbol{O}}$ & $1,77 \cdot 10^{5}$ & - & 88,68 \\
\hline $\boldsymbol{K}_{\boldsymbol{H}_{\mathbf{2}}}$ & $6,12 \cdot 10^{-8}$ & $\mathrm{MPa}^{-1}$ & $-82,90$ \\
\hline $\boldsymbol{P}_{\boldsymbol{H}_{\mathbf{2}}}$ & $2,19 \cdot 10^{-5}$ & $\mathrm{~mol} \cdot \mathrm{m}^{-1} \cdot \mathrm{s}^{-1} \cdot \mathrm{Pa}^{-0,5}$ & $-29,73$ \\
\hline
\end{tabular}

Para o cálculo da permeação de hidrogênio, uma zona de permeação isobárica e o arraste instantâneo do hidrogênio permeado foram adotadas como hipóteses iniciais. O valor permeado de hidrogênio foi adicionado no termo fonte, $S_{m}$, promovendo um consumo do hidrogênio na zona de reação e geração de hidrogênio na zona de arraste. Outras formas de implementação do termo de permeação estão sendo estudadas com o intuito de estudar diferentes fenômenos no equilíbrio termodinâmico.

\subsection{ESTUDO DE CONVERGÊNCIA DE MALHA}

Para fins de análise da convergência da malha a ser utilizada foi aplicado o estudo relatado por Roache [12]. Inicialmente, foram geradas cinco malhas, com diferentes números de elementos, para analisar a influência da mesma em duas variáveis de interesse, a variação da quantidade de metano na zona de reação e a variação da quantidade de hidrogênio na zona de arraste.

A metodologia proposta por Roache [12] está resumida nas seguintes etapas: 
Etapa 1 - Estabelecer uma célula representativa, a partir da Equação (21), para a realização posterior dos cálculos tridimensionais.

$h=\left[\frac{1}{N} \sum_{i=1}^{N} V_{i}\right]^{\frac{1}{3}}$

onde $V_{i}$ é o volume de cada elemento da malha estudada e $\mathrm{N}$ é o número de elementos da mesma.

Etapa 2 - Escolher diferentes refinos de malha, utilizando como critério a razão entre os refinos, $r=\frac{r_{\text {grosseiro }}}{r_{\text {refinado }}}$, seja maior que 1,3 (valor baseado em estudos empíricos). A sequência de refinos deve ser: $h_{1}<h_{2}<h_{3}<h_{4}<h_{5}$.

Etapa 3 - Considerando a análise das malhas 1 e 2, calcula-se os parâmetros de ordem da malha utilizando as Equações (22a), (22b) e (22c).

$$
\begin{aligned}
& p=\frac{1}{\ln \left(r_{21}\right)}|\ln | \frac{e_{32}}{e_{21}}|+q(p)| \\
& q(p)=\ln \left(\frac{r_{21}^{p}-s}{r_{32}^{p}-s}\right) \\
& s=1 \cdot \operatorname{sqn}\left(\frac{e_{32}}{e_{21}}\right)
\end{aligned}
$$

sendo, $e_{32}=\emptyset_{3}-\emptyset_{2}, e_{21}=\emptyset_{2}-\emptyset_{1}$ com $\emptyset$ sendo o ponto na malha que se deseja analisar a variação das variáveis estudadas de acordo com o refino de malha utilizado. Neste trabalho, foi analisada a concentração molar de metano no meio reacional e a concentração molar de hidrogênio na região de arraste.

Etapa 4 - Calcular os valores extrapolados a partir da Equação (23).

$$
\emptyset_{\text {ext }}^{21}=\frac{\left(r_{21}^{p} \phi_{1}-\emptyset_{2}\right)}{\left(r_{21}^{p}-1\right)}
$$

Etapa 5 - Calcular o erro aproximado e extrapolado entre as malhas e o índice de convergência de malha (GCI) a partir das Equações (24), (25) e (26), respectivamente.

$$
\begin{aligned}
& e_{a}^{21}=\left|\frac{\emptyset_{1}-\emptyset_{2}}{\emptyset_{1}}\right| \\
& e_{e x t}^{21}=\frac{\emptyset_{e x t}^{21}-\phi_{1}}{\emptyset_{e x t}^{21}} \\
& G C I_{21}=\frac{1,25 e_{a}^{21}}{r_{21}^{p}-1}
\end{aligned}
$$

As etapas descritas acima foram repetidas para a análise e comparação dos outros refinos das malhas estudadas. Tais etapas foram executados por meio de uma subrotina criada no software Scilab.

\subsection{ESTUDO DE OTIMIZAÇÃO E APLICAÇÃO DOS MÉTODOS HEURÍSTICOS}

A otimização realizada teve como função objetivo a produtividade do processo, calculada como sendo a razão entre quantidade de hidrogênio permeada e quantidade alimentada de metano (Equação 20), função esta a ser maximizada. As variáveis do processo a serem otimizadas foram pressão operacional, temperatura do reator e razão molar de alimentação entre água e metano. $\mathrm{O}$ critério de seleção das variáveis a serem otimizadas foi baseado em trabalhos encontrados na literatura, os quais apresentam tais variáveis como influentes na eficiência do processo de reforma.

Para a busca das melhores condições para o processo, foi utilizado o método heurístico AG, o qual foi desenvolvido em linguagem de programação FORTRAN 90. Os códigos foram adaptados do trabalho de Santos et al. [15] e Carrol [12], de modo que fosse possível integrar o algoritmo ao simulador. 
Primeiramente, o algoritmo ordena ao CFX que execute a simulação escolhendo aleatoriamente as variáveis do processo (chute inicial) e guarda o resultado obtido para a função objetivo e os valores dessas variáveis, adotando as estratégias intrínsecas do AG. A cada iteração, novos valores são inseridos para a simulação e estes são avaliados, caso o valor da função seja maior que o anterior ele será guardado pelo algoritmo. Com isso, é realizada uma varredura no espaço de busca definido, com base nos critérios do método em questão. No final da execução, o algoritmo terá guardado o valor máximo da função objetivo. A quantidade de iterações necessárias deve ser definida pelo usuário conforme a sua necessidade. A Figura 2 ilustra o esquema da estratégia de otimização que integra o ANSYS/CFX ${ }^{\circledR}$ e o código de otmização desenvolvido em Fortran e a Figura 3 mostra um fluxograma genérico de como foi implementado o AG.

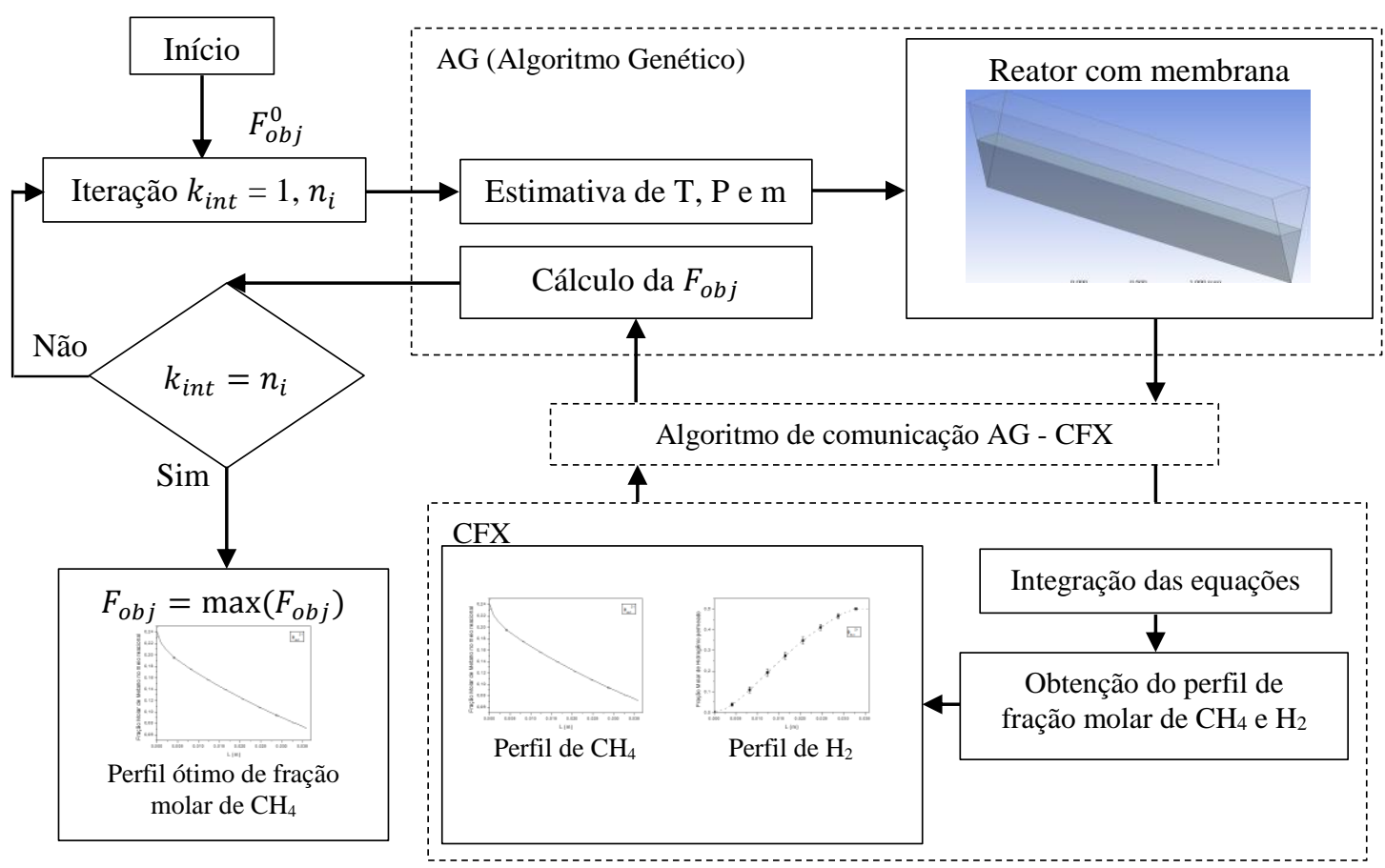

Figura 2: Esquema da interface de comunicação Fortran/CFX ${ }^{\circledR}$

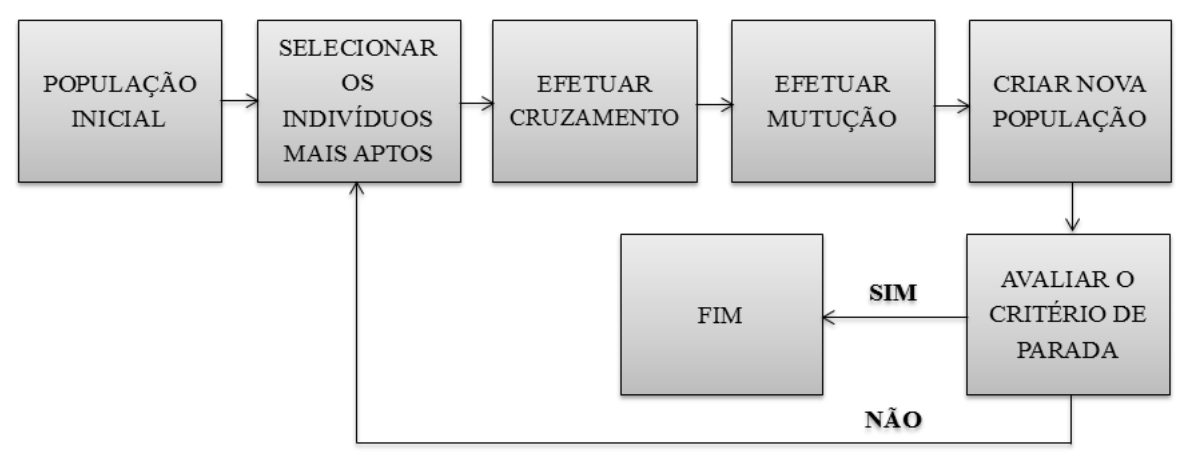

Figura 3: Fluxograma genérico para implementação do $A G$

$\mathrm{Na}$ Tabela 3 encontram-se os valores dos parâmetros utilizados no AG e os valores dos limites de busca de cada variável do processo a ser otimizado. Vale salientar que estes valores são escolhidos com base na função objetivo analisada e também considerando valores reportados na literatura. 
Tabela 3: Valores dos operadores e parâmetros utilizados nos algoritmos de busca e limites de busca das variáveis do processo utilizadas na otimização

\begin{tabular}{ccc}
\hline \multicolumn{2}{c}{ Número de Indivíduos } & 100 \\
\hline \multicolumn{2}{c}{ Número de Gerações } & 26 \\
\hline \multicolumn{2}{c}{ Número de dimensões } & 3 \\
\hline \multicolumn{2}{c}{ Probabilidade de Mutação } & 0,01 \\
\hline \multicolumn{2}{c}{ Probabilidade de Cruzamento } & 0,50 \\
\hline \multicolumn{2}{c}{ Limite inferior } & Limite superior \\
\hline $\begin{array}{c}\text { Pressão operacional } \\
(\mathbf{k P a})\end{array}$ & 102,00 & 506,63 \\
\hline $\begin{array}{c}\text { Temperatura do } \\
\text { reator }(\mathbf{K})\end{array}$ & 573,15 & 873,15 \\
\hline $\begin{array}{c}\text { Razão molar de } \\
\text { alimentação } \mathbf{H}_{\mathbf{2}} \mathbf{O} / \mathbf{C H}_{4}\end{array}$ & 2,50 & 6,00 \\
\hline
\end{tabular}

\section{RESULTADOS E DISCUSSÃO}

\subsection{ANÁLISE DE CONVERGÊNCIA DE MALHA}

A metodologia proposta por Roache [12] foi aplicada para a construção dos diferentes refinos de malha para o reator com membrana. Para isto, geraram-se 5 malhas com diferentes números de elementos, a saber, 1944, 980, 480, 209 e 104 elementos, denominadas respectivamente de M1, M2, M3, M4 e M5.

Para analisar a influência das malhas nos resultados obtidos nas simulações foram consideradas a concentração molar de metano ao longo da zona de reação e a concentração molar de hidrogênio recuperado na zona de arraste, visto a importância dessas variáveis para o processo. O estudo foi realizado em 36 pontos ao longo da zona de reação e zona de arraste, a fim de se verificar a incerteza dos valores encontrados.

A Figura 4 apresenta os perfis de concentração molar de hidrogênio e metano ao longo do reator, mostrando os resultados obtidos nas simulações com as diferentes malhas utilizadas neste estudo.
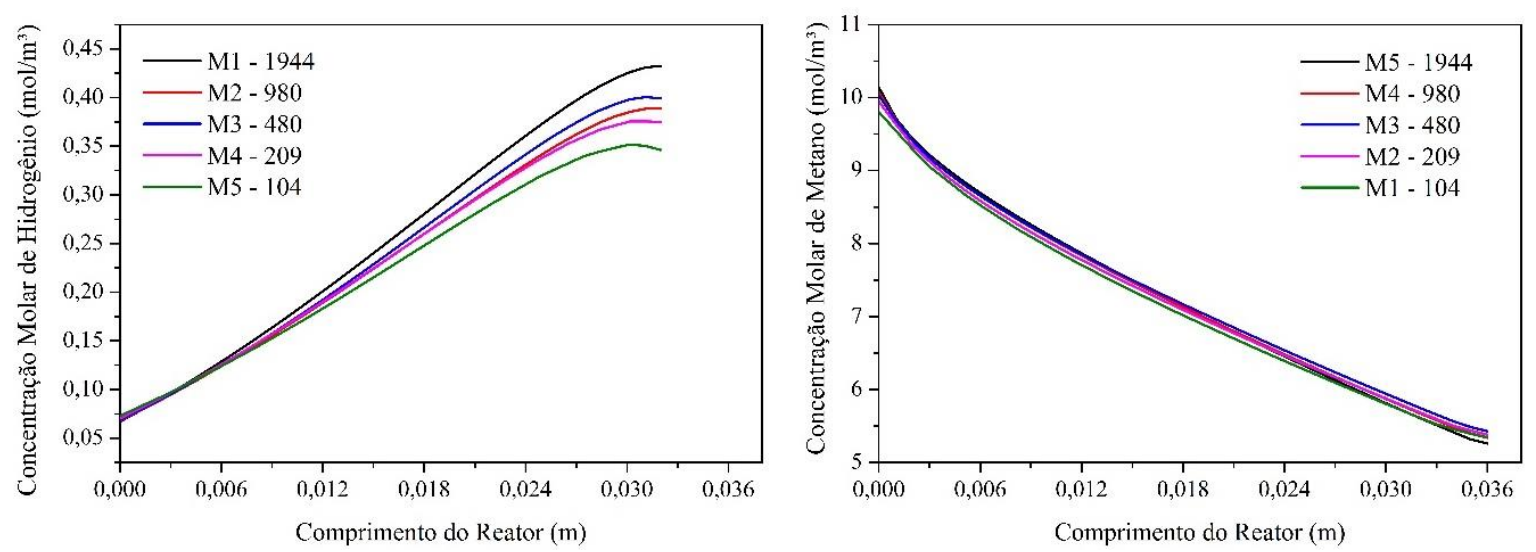

Figura 4: Perfis de concentração molar de hidrogênio na zona de arraste e perfis de concentração molar de metano na zona de reação

Como resultado da análise de convergência de malha, foram obtidos os valores de índice de convergência de malha (GCI) mínimos, máximos e médios entre as malhas para cada variável estudada. Os valores de GCI obtidos na análise da concentração de hidrogênio e concentração de metano estão dispostos, respectivamente, nas Tabelas 4 e 5. 
Tabela 4: Valores de GCI mínimos, máximos e médios entre as malhas para a concentração molar de hidrogênio na zona de arraste

\begin{tabular}{lccc}
\hline & Mínimo & Máximo & Médio \\
\hline GCI $_{\mathbf{1 1}}$ & 0,0059 & 0,2192 & 0,1336 \\
\hline GCI $_{\mathbf{3 2}}$ & 0,0160 & 0,0691 & 0,0436 \\
\hline GCI $_{\mathbf{4 3}}$ & 0,0014 & 0,1047 & 0,0440 \\
\hline GCI $_{54}$ & 0,0017 & 0,1618 & 0,0845 \\
\hline
\end{tabular}

Tabela 5: Valores de GCI mínimos, máximos e médio entre as malhas para a concentração molar de metano na zona de reação

\begin{tabular}{lccc}
\hline & Mínimo & Máximo & Médio \\
\hline $\mathbf{G C I}_{21}$ & 0,0004 & 0,0344 & 0,0119 \\
\hline $\mathbf{G C I}_{\mathbf{3 2}}$ & 0,0000 & 0,0328 & 0,0128 \\
\hline $\mathbf{G C I}_{43}$ & 0,0084 & 0,0186 & 0,0162 \\
\hline GCI $_{54}$ & 0,0115 & 0,0301 & 0,0209 \\
\hline
\end{tabular}

A partir dos valores de GCI listados nas tabelas acima, pode-se observar que os mais baixos valores entre as malhas para a variável na zona de arraste está situado entre as malhas 3 e 2 . Já para a variável da zona de reação foi observado que os menores valores de GCI médio foram entre as malhas 2 e 1 , porém os menores GCI mínimo e máximo foram entre as malhas 3 e 2 . Contudo, deve-se observar que os valores obtidos para o GCI estão muito próximos, para se afirmar uma diferença significativa somente se considerando os valores absolutos.

Com o intuito de complementar o estudo de convergência de malha proposto por Roache [12], foi considerado o tempo que o software ANSYS/CFX ${ }^{\circledR}$ levou para solucionar o problema proposto, analisando conjuntamente os valores de critério de convergência RMS para as equações de massa, movimento e turbulência e estes deveriam apresentar um valor menor que $10^{-5}$. A malha M3, de 480 elementos, foi a que concluiu o problema proposto num tempo computacional menor, levando 1 minuto e 15 segundos para encontrar os resultados da simulação.

Dessa forma, levando em consideração a análise dos resultados obtidos através do método proposto por Roache [12], percebe-se que os erros associados a cada refino de malha são pequenos frente aos valores de concentração calculados. Neste cenário, considerando ainda o tempo computacional gasto para a resolução do problema, a malha 3 foi adotada como sendo adequada para o desenvolvimento das próximas etapas do trabalho. A Figura 5 apresenta a malha utilizando na modelagem, simulação e otimização do problema.

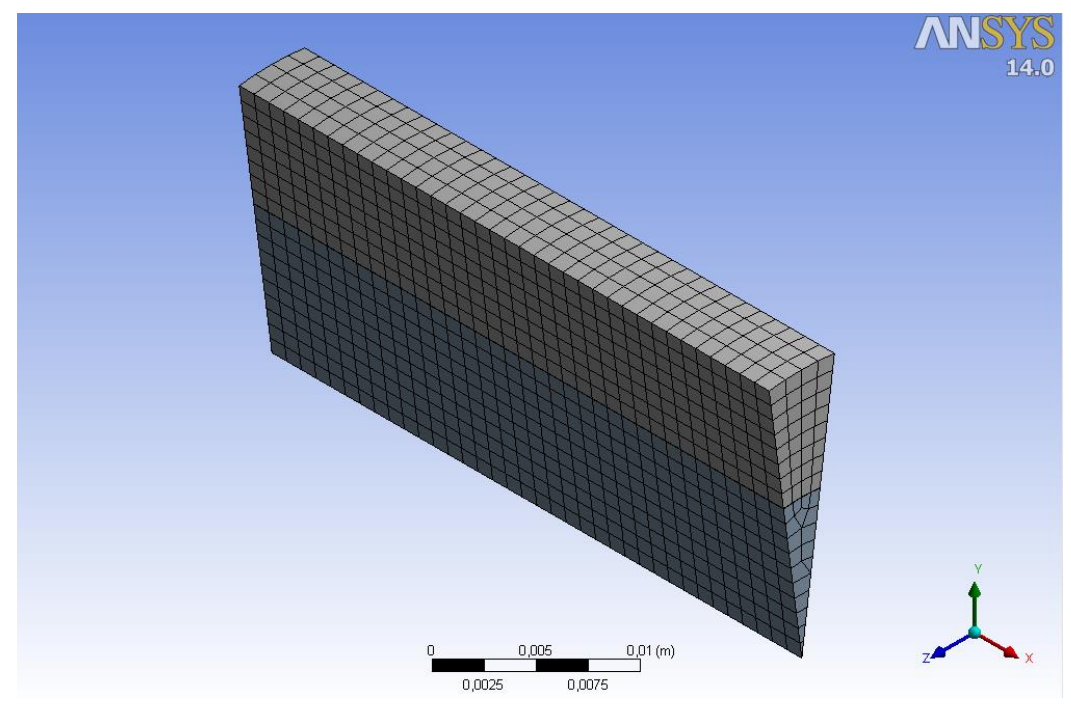

Figura 5: Malha com 480 elementos 


\subsection{VALIDAÇÃO DA SIMULAÇÃO}

A validação do reator com membrana foi realizada comparando os dados obtidos na simulação com dados experimentais apresentados por Shu et al. [10]. No trabalho citado, foi realizado um estudo do efeito de algumas variáveis como temperatura do reator e pressão do reator na conversão de metano de um reator com membrana de paládio suportada em aço inoxidável.

Os valores obtidos a partir dos dados de conversão obtidos no trabalho de Shu et al. [10] e os resultados obtidos nas simulações realizadas são mostrados na Figura 6. A Figura 6(A) mostra a conversão de metano em função da temperatura do reator, com variação entre 573,15 e 773,15 K, mantendo a pressão de operação de $136 \mathrm{kPa}$ e razão molar de alimentação igual a 3 . Na Figura 6(B) pode-se visualizar os valores experimentais e simulados da conversão de metano, variando a pressão operacional do reator a valores entre 146 e $200 \mathrm{kPa}$, mantendo-se a temperatura do reator a $773,15 \mathrm{~K}$ e razão de alimentação igual a 3.

Os valores simulados, variando-se a temperatura do reator, estão próximos dos valores experimentais encontrados por Shu et al. [10]. Houve uma diferença máxima de aproximadamente $11 \%$ na temperatura de $573,15 \mathrm{~K}$, no entanto, essa diferença foi diminuindo com o aumento da temperatura, chegando a uma diferença de aproximadamente $0,5 \%$ na temperatura de $773,15 \mathrm{~K}$. No caso da variação da pressão operacional, os dados empíricos e simulados foram próximos em toda a faixa de pressão analisada, apresentando valor muito similar para a pressão de $200 \mathrm{kPa}$ e uma diferença de aproximadamente $3 \%$ na pressão de $146 \mathrm{kPa}$.
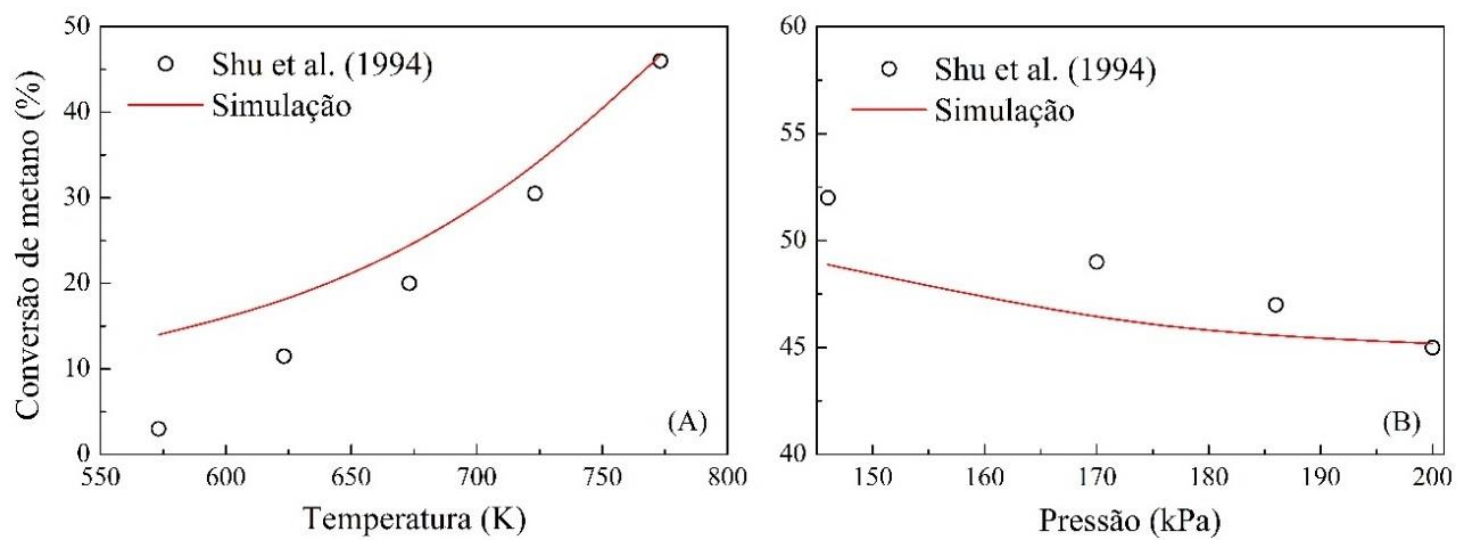

Figura 6: Valores empíricos reportados por Shu et al. [10] e simulados de conversão de metano em função da temperatura $(A)$ e pressão $(B)$ do reator, respectivamente

Com base nestes resultados, a validação é considerada eficiente no que se refere a descrever os resultados experimentais relatados na literatura. Portanto, o modelo simulado pode ser aplicado em estudos de otimização, como o que foi desenvolvido neste trabalho.

\subsection{OTIMIZAÇÃO DO PROCESSO}

Para obtenção de perfis operacionais ótimos, foi aplicado o AG com o intuito de maximizar a função objetivo. Para isso, o código foi executado cinco vezes, sendo que, em cada execução, eram realizadas 2600 iterações, número este obtido pela multiplicação entre o número de indivíduos e o número de gerações escolhidas. Ressalta-se que os valores dos parâmetros do algoritmo foram determinados de acordo com Carrol [16].

$\mathrm{O}$ valor ótimo obtido para a função objetivo produtividade foi de $1,28 \mathrm{~kg}_{\mathrm{H} 2} \cdot \mathrm{kg}_{\mathrm{CH}}{ }^{-1}$. Este valor foi encontrado com as condições operacionais de $102 \mathrm{kPa}, 873,08 \mathrm{~K}$ e uma razão molar de alimentação igual a 6,00; os mesmos valores foram obtidos em todas as execuções. Para comprovar a validade dos resultados obtidos foram avaliados os valores da quantidade de metano e hidrogênio ao longo do reator, conforme as Figuras 7 e 8.

Pode-se observar que variação da quantidade de metano, mostrada na Figura 7 , indica que todo o metano inserido no reator foi convertido, obtendo uma conversão de metano de $100 \%$. O mesmo percentual foi obtido para a recuperação de hidrogênio, pois, como se pode visualizar na 
Figura 8, todo o hidrogênio produzido foi permeado através da membrana para a zona de arraste. Silva et al. [17] obteve resultados similares na otimização do mesmo problema utilizando o algoritmo DIRCOL escrito em linguagem FORTRAN, no qual foi alcançada uma taxa de conversão de metano de $96 \%$ e uma taxa de $91 \%$ de recuperação de hidrogênio. O mesmo autor também aponta uma série de trabalhos na literatura com resultados similares para conversão de metano.

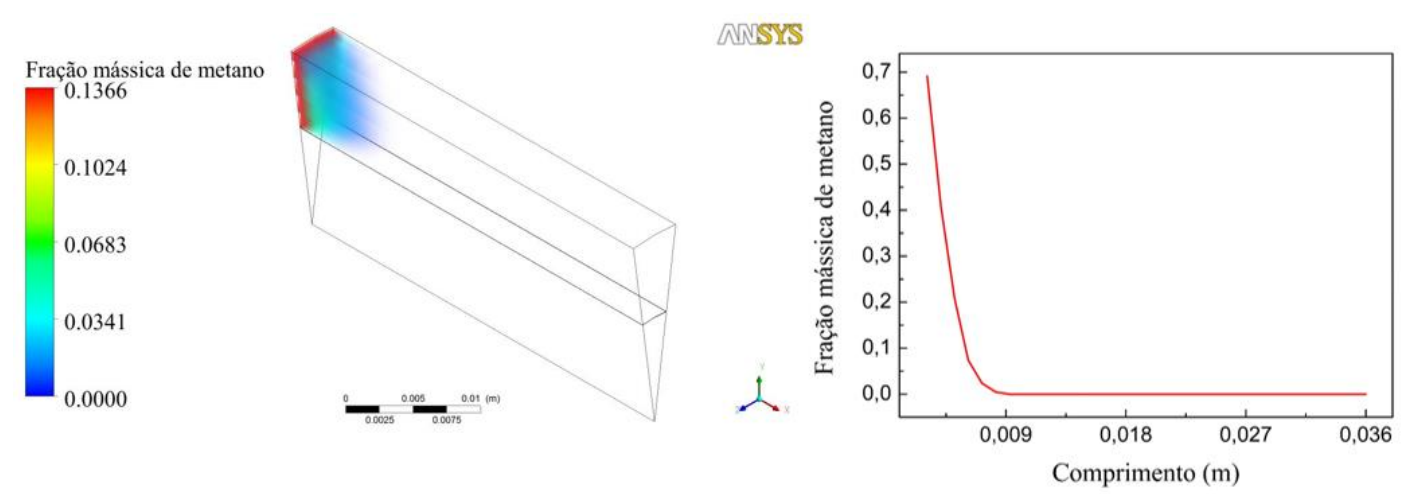

Figura 7: Variação da quantidade mássica de metano ao longo do reator com membrana com as condições otimizadas
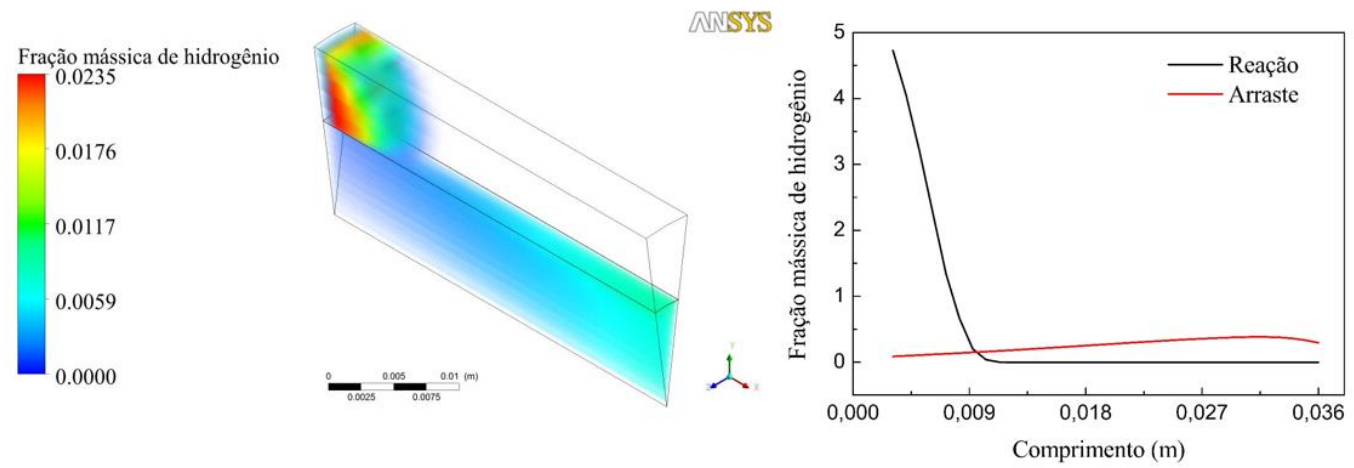

Figura 8: Variação da quantidade mássica de Hidrogênio ao longo do reator com membrana com as condições otimizadas

É válido salientar que os gráficos apresentados nas Figuras 7 e 8 indicam valores de fração mássica. Por isso, os dados da fração de mássica de hidrogênio na entrada da zona de reação e na saída da zona e arraste não podem ser comparados, pois se tratam de domínios diferentes, contudo, percebe-se que a metodologia aplicada proporcionou melhores condições operacionais que as encontradas na literatura. Deve-se destacar que esta metodologia integrou o código de otimização a um programa comercial de simulação, determinando condições ótimas de operação. Além disso, a adaptação do procedimento apresentado para diferentes processos químicos pode ser realizada sem maiores dificuldades.

No entanto, a simulação realizada ainda está sendo aperfeiçoada, pois as equações de transferência de massa através da membrana estão sendo aprimoradas para obtenção dos valores mais realísticos da permeação de hidrogênio ao longo da membrana.

\section{CONCLUSÃO}

Considerando os resultados apresentados, pode-se concluir que a simulação realizada forneceu resultados coerentes com o processo estudado, apresentando valores próximos aos descritos na literatura. As validações apresentadas na Figura 5 mostraram que no estudo avaliando o efeito da temperatura na conversão de metano houve uma diferença máxima de aproximadamente $11 \%$ no valor de conversão simulado na temperatura de $573,15 \mathrm{~K}$, no entanto, essa diferença foi diminuindo com o aumento da temperatura, chegando a uma diferença de aproximadamente 
0,5\% para o valor da conversão na temperatura de $773,15 \mathrm{~K}$. Ainda, o efeito da pressão na conversão do metano apresentou um erro máximo de $3 \%$ na pressão de $146 \mathrm{kPa}$ e um erro mínimo de $0,4 \%$ a $200 \mathrm{kPa}$, corroborando os resultados simulados neste trabalho para a conversão de metano. Portanto, pode-se afirmar que a abordagem adotada descreveu satisfatoriamente o reator com membrana proposto no trabalho de Shu et al. [10].

No que se refere ao estudo de otimização, notou-se que os resultados obtidos estão coerentes com os resultados apresentados na literatura, apresentando resultados que conduzem conversões superiores às apresentadas na literatura. A implementação do método heurístico integrado ao simulador comercial para maximizar a função objetivo conduziu a um valor de produtividade do processo de $1,28 \mathrm{~kg}_{\mathrm{H} 2} \cdot \mathrm{kg}_{\mathrm{CH} 4}{ }^{-1}$, além disso, as taxas de conversão de metano e recuperação de hidrogênio alcançaram valores próximos de $100 \%$. Adicionalmente, é importante destacar os bons resultados obtidos aplicando a otimização conjugada entre o método de busca e o simulador comercial, sendo este simulador utilizado para o computo da função objetivo, uma das etapas da abordagem apresentada. Além disso, deve ser destacada a originalidade do trabalho visto que a integração entre o Algoritmo Genético e software comercial vem sendo pouco explorada na literatura.

\section{AGRADECIMENTOS}

Os autores gostariam de agradecer ao CNPq (Projeto UNIVERSAL - Processo 484493/20124), FATIPEC/SE (Projeto PRONEM - EDITAL FAPITEC/SE/FUNTEC/CNPq N ${ }^{\circ}$ 10/2011 e Projeto PPP - EDITAL FAPITEC / SE / FUNTEC / CNPq N 04/2011) e ao PEQ/UFS pelo suporte financeiro e colaboração.

\section{REFERÊNCIAS BIBLIOGRÁFICAS}

1. Nicodemos RM, Lima AM, Assis AJ. Produção de Hidrogênio a partir de fontes renováveis. Horizonte Científico. 2011 Jul;5(1).

2. Kyriakide AS, Garcia LR, Ipsakis D, Seferlis P, Papadopoulou S. Enhancement of pure hydrogen production through the use of a membrane reactor. Int J Hydrogen Energ. 2014 Mar 18;39(9):467-491, doi:10.1016/j.ijhydene.2014.01.093.

3. Martínez I, Romano MC, Fernández JR, Chiesa P, Murillo R, Abanades JC. Process design of a hydrogen production plant from natural gas with $\mathrm{CO} 2$ capture based on a novel $\mathrm{Ca} / \mathrm{Cu}$ chemical loop. Appl Energ. 2014 Fev;114:192-208, doi:10.1016/j.apenergy.2013.09.026.

4. Smith RB, Muruganandam L, Shekar SM. CFD Analysis of Water Gas Shift Membrane Reactor. Chem Eng Res Des. 2011 Nov;89(11):2448-2456, doi:10.1016/j.cherd.2011.02.031.

5. Reeves CR. Genetic Algorithms In: Gendreau M, Potvin J-Y, editors. Handbook of Metaheuristics. New York: Springer, 2010, 649p.

6. Libório DO, Santos EJ, Santana PL, Silva CF, Silva AS. Uma base preliminar de informações para o estudo do movimento de uma suspensão particulada com restrição de incompressibilidade. Sci Plena. 2015 Set 16; 11(10), doi: 10.14808/sci.plena.2015.104202.

7. Silva AS, de Jesus E, Pagano RL, Meneses LDM, Rocha CPS. Equações do movimento de suspensões particuladas com restrição de incompressibilidade. Sci Plena. 2013 Nov 01; 9(11), doi: 10.14808/sci.plena.2013.114201.

8. Silva AS, Santana PL, Pagano RL, Jesus E, Rocha CPS. Caracterização de suspensões floculentas com base em modelagem matemática da sedimentação em proveta. Sci Plena. 2013 Mai 27; 9(5), doi: 10.14808/sci.plena.2013.054201.

9. Santos LS, Pagano RL, Calado VMA, Biscaia Jr. EC. Optimization of a pultrusion process using finite difference and particle swarm algorithms. Braz J Chem Eng. 2015 Jun; 32(2):543-553, doi: 10.1590/0104-6632.20150322s00003181

10. Shu J, Grandjean BA, Kaliaguine S, Aliaguine S. Methane steam reforming in asymmetric Pd- and PdAg/porous SS membrane reactors. Appl Catal A-Gen. 1994 Ago 2;119(2):305-325, doi:10.1016/0926860X(94)85199-9.

11. Xu J, Froment GF. Methane Steam Reforming, Methanation and Water-Gas Shift: I. Intrinsic Kinetics. AIChe Jo. 1989 Jan;35(1):88-96, doi:10.1002/aic.690350109.

12. Roache PJ. Perspective: A Method for Uniform Reporting of Grid Refinement Studies. J. Fluids Eng. 1994 Set;116(3):405-413, doi: 10.1115/1.2910291. 
13. Bathe KJ. Finite Element Procedures. $2^{\mathrm{a}}$ ed. New Jersey:Prentice-Hall; 2007. 1038p.

14. Reichelt W., Blasz E. Strömungstechnische Untersuchungen an mit Raschig-Ringen gefüllten Füllkörperrohren und-säulen. Teil I: Einphasen-Gasströmung. Chem-Ing-Tech. 1971 Set; 43(17):949956, doi: 10.1002/cite.330431702.

15. Santos LS, Biscaia Jr EC, Pagano, RL, Calado, VMA. CFD-optimization algorithm to optimize the energy transport in pultruded polymer composites. Braz J Chem Eng. 2012 Fev; 29(03):559-566, doi: 10.1590/S0104-66322012000300013.

16. Carrol DL. Genetic. Algorithm. 1998. Disponível em: <http://jblevins.org/mirror/amiller/>. Acesso Em: 4 de outubro de 2015.

17. Silva LC, Murata VV, Hori CE, Assis AJ. Hydrogen production from methane steam reforming: parametric and gradient based optimization of a Pd-based membrane reactor. Optim Eng. $2010 \mathrm{Fev}$ 19;11(3):441-458, doi: 10.1007/s11081-010-9106-2. 